\section{A safety audit of the first 10000 intravitreal ranibizumab injections performed by nurse practitioners}

\begin{abstract}
Purpose To evaluate the safety of a nurse practitioner (NP)-delivered injection service for the treatment of wet age-related macular degeneration (wAMD) with ranibizumab. Methods An evaluation of medical staffing resources for providing an injection service for wAMD highlighted difficulties covering lists. An alternative strategy of an NP-delivered injection service was evaluated. Two suitable NPs with previous extensive experience in minor surgical procedures were identified. The department's senior vitreoretinal consultant supervised the NP's training programme. A prospective safety audit was conducted for the first 5.5 years of the service.

Results The NPs administered 10006 injections in the first 5.5 years of the service (1 May 2008 to 8 October 2013). This represented $84.1 \%$ of the total injections performed during this period. Four patients developed presumed infectious endophthalmitis ( 1 was culture positive and 3 were culture negative). The incidence of post-

\section{Introduction}

Following publication of the pivotal MARINA ${ }^{1}$ and $\mathrm{ANCHOR}^{2}$ trials the National Institute for Health and Clinical Excellence (NICE) technology appraisal guidance TA155 recommended intravitreal injection of the anti-vascular endothelial growth factor (anti-VEGF) agent ranibizumab, for wet age-related macular degeneration (wAMD) treatment in the National Health Service (NHS). The annual incidence of wAMD requiring antiVEGF treatments has been estimated at $\sim 26000$ patients in the United Kingdom (UK), ${ }^{3}$ but this may be an underestimate. ${ }^{4}$ The West of England Eye Unit (WEEU) based at the Royal Devon and Exeter NHS Foundation Trust (RD\&E), Exeter, serves a population of $\sim 350000$ patients and therefore expects 150 new cases of wAMD per year. The existing resources at WEEU were felt insufficient to cope with the service demands of this number of patients requiring treatments according to the PrONTO study $y^{5,6}$ and new methods of service delivery were sought.
\end{abstract} injection endophthalmitis was $0.04 \%$. There was no evidence of lens touch, retinal detachment, or systemic thrombo-embolic events.

Conclusions Carefully selected and welltrained NPs are capable of delivering a safe and effective wAMD injection treatment service. This work demonstrates how such a service can be established and provides safety data that other units can use as a benchmark when evaluating their own practice.

Eye (2014) 28, 1161-1164; doi:10.1038/eye.2014.153; published online 18 July 2014
Materials and methods

\section{Resource needs}

The two key resource constraints were lack of personnel and space for the injections within the department. Intravitreal injections had hitherto formed part of routine ophthalmic operating lists. A business plan was formulated for the provision of a room near the out-patient facility dedicated to intravitreal injections. This would accommodate the proposed new wAMD

P Simcock, B Kingett, N Mann, V Reddy and J Park

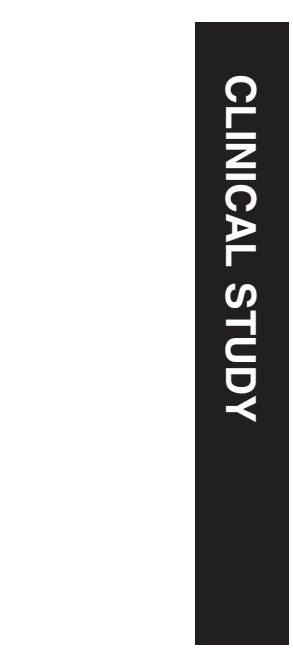

West of England Eye Unit, Royal Devon \& Exeter NHS Foundation Trust, Exeter EX2 5DW, UK

Correspondence: P Simcock, West of England Eye Unit, Royal Devon \& Exeter NHS Foundation Trust, Barrack Road, Exeter EX2 5DW, UK Tel: + 441392406008 ; Fax: + 441392406002 E-mail: peter.simcock@ nhs.net

Received: 14 March 2014 Accepted: 22 May 2014 Published online: 18 July 2014

This work has been presented in oral format at the British and Eire Association of Vitreoretinal Surgeons meeting in Bristol in November 2013 (with the current data of 10006 injections) and as a poster presentation at the Royal College of Ophthalmologist Annual Congress in May 2013 (with 6383 injections). 
treatment pathway while freeing up existing theatre lists, thus providing a dual benefit.

The Clinical Governance committee (CGC) of the RD\&E felt that 'to meet the anticipated demand (for intravitreal ranibuzimab injections), WEEU would need to provide a minimum of 4 sessions per week of treatments, coupled with additional outpatient sessions and investigations' (Minutes of RD\&E Governance Committee Meeting, 10 April 2008). As there was no capacity or finance available to increase the medical staffing in the WEEU, it decided on behalf of the trust 'to accept vicarious liability' for a suitably qualified and experienced nurse practitioner (NP) to provide the service (Minutes of RD\&E Governance Committee Meeting, 10 April 2008).

A registered NP (BK) with previous training in the provision of minor lid surgery and sub-Tenon's anaesthesia and then, as the service expanded, a second NP (NM) were selected to be trained, supervised, and assessed in safe and effective intravitreal injection technique on a one-to-one basis by the senior vitreoretinal surgeon in the unit (PS).

\section{Hospital approval}

The CGC stipulated that 'only the identified NP was to carry out the injections, a Consultant/Specialist Registrar was always to be present in the WEEU for immediate supervision and advice if needed and an audit be undertaken to assess performance and safety issues' (RD\&E General Risk Assessment Record: intravitreal injection of Lucentis, 13 March 2008). The consent form specified that the NP, not an ophthalmologist, would be administering the injection. The NP was to commence independent practice only after completing at least 20 injections supervised by the trainer, PS (RD\&E Key Skills: intra-vitreal injection of Lucentis). The injection procedure is described in Box 1.

This audit was based on safety issues and NP practice was regularly reviewed (at least 6 monthly) to quantify complication rates to ensure NP practice continually compared favourably to audit standards, with specific reference to the incidence of endophthalmitis relative to the national average estimated by a previous study completed in association with the British Ophthalmic Surveillance Unit. ${ }^{7}$

\section{Results}

\section{Number of injections}

In the first 5.5 years of the service (1 May 2008-8 October 2013), 11893 injections of ranibizumab were given, of which $10006(84.1 \%)$ were administered by the NPs. The
Box 1 Procedure for ranibizumab injections by the NP

- Check visual acuity is within the criteria for treatment.

- Seek medical advice for the following contraindications:

- Recent evidence of conjunctivitis (eg red eye, sticky discharge), blepharitis (eg inflamed eyelids)

- Uncontrolled high blood pressure

- Unstable angina

- Recent stroke

- Local anaesthetic drops administered: Proxymetacaine $0.5 \%$, then Benoxinate $0.4 \%$.

- Eye washed with povidone-iodine $5 \%$ solution and sterile eye drape applied.

- Lid speculum inserted and patient asked to gaze to the left or right.

- Injection site measured: $4.0 \mathrm{~mm}$ from limbus for phakic and $3.5 \mathrm{~mm}$ from limbus for pseudo-phakic eyes.

- Intra-vitreal injection of $0.05 \mathrm{ml}$ of ranibizumab.

- Topical stat dose of ofloxacin.

- Test visual acuity for hand movements and enquire about onset of severe pain. Urgent medical assessment to be sought for significant loss of vision or severe pain.

proportion of injections given by the NPs has continued to increase with almost an entirely NP-delivered service in recent months.

\section{Complications}

Endophthalmitis There were four cases of presumed infectious endophthalmitis (one was culture positive and three were culture negative), as described:

Case 1: A 79-year-old male developed blurred vision 7 days after the injection. Treatment comprised a vitreous tap with intravitreal ceftazidime and vancomycin. Staphylococcus hominis was cultured. His visual acuity 1 year following the episode of endophthalmitis was 0.9 LogMAR (compared to 0.6 LogMAR pre-injection).

Case 2: A 61-year-old male presented with blurring of vision 4 days after the injection. Vision reduced from 0.62 LogMAR pre-injection to count fingers. No organisms were isolated from the vitreous tap and the same intravitreal treatment was given as in case 1 . The situation improved and current vision is 0.42 LogMAR, having required two further injections of ranibizumab.

Case 3: An 83-year-old lady received her 15th intravitreal injection. Six days later vision had deteriorated to hand movements, from $0.34 \log$ MAR pre-injection. The patient was immediately treated with a vitreous tap and intravitreal antibiotics (vancomycin and 
ceftazidime). The vitreous tap was culture negative. Two months later vision remains hand movements due to a dense cataract and persistent vitreous debris. During this time the patient has also experienced progressive dementia and moved to a residential home.

Case 4: A 91-year-old lady received her 19th intravitreal injection to her right eye, at the same time as an intravitreal injection to the left eye. In addition to wAMD, her past ophthalmic history included cataract surgery and primary open-angle glaucoma. Two days later the right eye became blurred and painful. The vision reduced to hand movements (relative to pre-injection acuity $0.9 \log$ MAR) and there was a 1-mm hypopyon. The left eye showed no signs of infection. The patient was immediately treated with anterior chamber and vitreous taps, and intravitreal vancomycin and ceftazidime. The aqueous and vitreous samples were culture negative. In the following 6 months the right eye improved and received three further intravitreal injections, and acuity is now at the pre-endophthalmitis level (0.86 logMAR).

Elevated intraocular pressure (IOP) One patient developed high IOP immediately following the intravitreal injection. This patient was a 77-year-old female who had eye discomfort and blurred vision immediately following injection. Her IOP was measured immediately and was raised at $38 \mathrm{~mm} \mathrm{Hg}$. Based on the injection protocol (Box 1), an ophthalmologist (PS) was called to attend the patient. The central retinal artery was pulsating and the IOP lowered medically, but a paracentesis needle was available if needed as part of an anterior chamber paracentesis kit that is in the injection room.

This episode demonstrated the robustness of the service-delivery model in its ability to manage potential ophthalmic emergencies (a trained ophthalmologist is always present in the WEEU when the NP is injecting and can be summoned immediately).

Other complications One patient developed a large subconjunctival haemorrhage.

There was no evidence of lens touch, retinal detachment, or systemic thrombo-embolic events in any of the patients.

\section{Discussion}

Endophthalmitis has been reported in large cohorts of patients injected with intravitreal ranibizumab. The MARINA study reported an incidence of $0.05 \%$ per injection, ${ }^{1}$ while the ANCHOR study reported $0.1 \% .^{2}$ The largest UK prospective study identified 47 cases through the British Ophthalmological Surveillance Unit over a 15-month period, but the denominator figure was extrapolated to the UK population from Scottish data and an estimated incidence of $0.025 \%$ was calculated. ${ }^{7}$ The four cases of endophthalmitis in this study represent an incidence of $0.04 \%$ for injections given by the NPs, which is favourable relative to the rates observed in the MARINA and ANCHOR studies and similar to that reported by the British Ophthalmological Surveillance Unit. Traumatic lens damage following ranibizumab injection has been reported, ${ }^{8}$ but no patients treated by the NP had evidence of inadvertent lens damage on follow-up medical review.

The West of England Eye Unit of the Royal Devon and Exeter Hospital is the first NHS Foundation Trust in the UK to develop a NP-delivered intravitreal injection service and has similar rates of adverse events to that reported in other large studies undertaken by ophthalmologists. An injection service provided by a carefully selected and rigorously trained NP adhering to the necessary clinical governance safeguards has the potential to help eye units throughout the UK to meet the rising demands for wAMD treatment in an ageing population. Research has demonstrated the benefit of anti-VEGF injections for 'centre-involved' diabetic macular oedema, ${ }^{9}$ central retinal vein occlusion, ${ }^{10}$ and branch retinal vein occlusion. ${ }^{11}$ NICE has recently approved the use of ranibizumab for diabetic macular oedema and retinal vein occlusions, and this will result in even more pressure on the resources of NHS eye units across the UK. NP-delivered anti-VEGF injections is one solution to assist in meeting these extra demands and this method of service delivery has recently been endorsed by the Royal College of Ophthalmologists (Intra-ocular injections by non-medical health care professionals, Royal College of Ophthalmologists statement, 01/05/2013) and the Macular Society.

This large safety audit of over 10000 injections demonstrates that carefully selected and well-trained NPs are capable of delivering a safe, effective wAMD injection treatment service, and provides a benchmark that other units can use when reviewing their own practice.

\section{Summary}

What was known before

- Anti-VEGF drugs are an effective treatment for wet AMD, diabetic macular oedema, and retinal vein occlusions.

- Anti-VEGF injections have a good safety profile when given by doctors; there is an ever-increasing number of patients requiring this treatment, and an ever-increasing demand on ophthalmologists to provide injections.

What this study adds

- Nurse practitioners can provide a safe and effective antiVEGF service, and can provide an new method of service delivery to allow ophthalmologists to concentrate on other aspects of eye care. 


\section{Conflict of interest}

The authors declare no confict of interest.

\section{References}

1 Rosenfeld PJ, Brown DM, Heier JS, Boyer DS, Kaiser PK, Chung CY et al. Ranibizumab for neovasculqar age-related macular degeneration. N Engl J Med 2006; 355: 1419-1431.

2 Brown DM, Kaiser PK, Michels M, Soubrane G, Heier JS, Kim RY et al. Ranibizumab versus Verteporfin for neovascular age-related macular degeneration. $N$ Engl J Med 2006; 355: 1432-1444.

3 Colquitt JL, Jones J, Tan SC, Takeda A, Clegg AJ, Price A. Ranibizumab and pegaptanib for the treatment of age-related macular degeneration: a systematic review and economic evaluation. Health Technol Assess 2008; 12 iii-iv ix-201.

4 Owen CG, Jarrar Z, Wormald R, Cook DG, Gletcher AE, Rudnicka AR. The estimated prevalence and incidence of late stage age-related macular degeneration in the UK. $\mathrm{Br} J$ Ophthalmol 2012; 96: 752-756.

5 Fung AE, Lalwani GA, Rosenfeld PJ, Dubovy SR, Michels S, Feuer WJ et al. An optical coherence tomography-guided, variable dosing regimen with intravitreal ranibizumab (Lucentis) for neovascular age-related macular degeneration. Am J Ophthalmol 2007; 143: 566-583.
6 Lalwani GA, Rosenfeld PJ, Fung AE, Dubovy SR, Michels S, Feuer $\mathrm{W}$ et al. A variable-dosing regimen with intravitreal ranibizumab for neovascular age-related macular degeneration: year 2 of the PrONTO Study. Am J Ophthalmol 2009; 148: 43-58

7 Lyall DAM, Tey A, Foot B, Roxburgh STD, Virdi M, Robertson $C$ et al. Post-intravitreal anti-VEGF endophthalmitis in the United Kingdom: incidence, features, risk factors, and outcomes. Eye 2012; 26: 1517-1526.

8 Saeed MU, Prasad S. Management of cataract caused by inadvertent capsule penetration during intravitreal injection of ranibizumab. J Cataract Refract Surg 2009; 35: 1857-1859.

9 Elman MJ, Aiello LP, Beck RW, Bressler NM, Bressler SB, Edwards AR et al. Randomized trial evaluating ranibizumab plus prompt or deferred laser or triamcinolone plus prompt laser for diabetic macular oedema. Ophthalmology 2010; 117: 1064-1077.

10 Brown DM, Campochiaro PA, Sing RP, Li Z, Gray S, Saroj $\mathrm{N}$ et al. Ranibizumab for macular edema following central retinal vein occlusion: six-month primary end point results of a phase III study. Ophthalmology 2010; 117: 1124-1133.

11 Campochiaro PA, Heier JS, Feiner L, Gray S, Saroj N, Rundle AC et al. Ranibizumab for macular edema following branch retinal vein occlusion: six-month primary end point results of a phase III study. Ophthalmology 2010; 117: 1102-1112. 